\title{
Erfassung der Anreicherung von Metallen und Stickstoff in baden-württembergischen Moosen
}

\author{
Winfried Schröder · Marcel Holy · Roland Pesch • Ilia Ilyin · Harry Harmens · Harald Gebhardt
}

Eingegangen: 5. Februar 2010/Akzeptiert: 8. Juni 2010/Online veröffentlicht: 8. Juli 2010

(C) Springer-Verlag 2010

\begin{abstract}
Zusammenfassung Hintergrund, Ziel und Bereich Umweltmonitoring ist zentraler Bestandteil der UNECE Nachhaltigkeitsstrategie. Hierbei dient das Moosmonitoring der Dauerbeobachtung der Anreicherung von Metallen in terrestrischen Ökosystemen und liefert seit 1990 alle fünf Jahre europaweit flächendeckende Inventurdaten. 2005 wurde auch die Stickstoffanreicherung erfasst. Das Moosmonitoring wird in Deutschland von Bund und Ländern gemeinsam durchgeführt. Gegenstand dieser Untersuchung ist die Kartierung der zeitlichen Entwicklung der Metallanreicherung in Baden-Württemberg seit 1990, die Stickstoffanreicherung 2005, die räumliche Varianz der Metallbioakkumulation in
\end{abstract}

W. Schröder $\cdot$ M. Holy $\cdot$ R. Pesch $(\square)$

Lehrstuhl für Landschaftsökologie, Hochschule Vechta, PF 1553, 46364 Vechta, Deutschland

E-Mail: rpesch@iuw.uni-vechta.de

M. Holy

E-Mail: mholy@iuw.uni-vechta.de

W. Schröder

E-Mail: wschroeder@iuw.uni-vechta.de

I. Ilyin

Meteorological Synthesizing Centre East of EMEP,

Krasina pereulok, 16/1, 123056 Moscow, Russia

E-Mail: ilia.ilyin@msceast.org

H. Harmens

Centre for Ecology and Hydrology, Environment Centre Wales,

Deiniol Road, Bangor, Gwynedd LL57 2UW, UK

E-Mail: hh@ceh.ac.uk

H. Gebhardt

Mitglied im Arbeitskreis Bioindikation und Wirkungsermittlung der Landesanstalten und -ämter, Landesanstalt für Umwelt,

Messungen und Naturschutz Baden-Württemberg,

Medienübergreifende Umweltbeobachtung, Klimawandel,

Griesbachstraße 1, 76185 Karlsruhe, Deutschland

E-Mail: harald.gebhardt@lubw.bwl.de
Abhängigkeit von Eigenschaften der Moosbeprobungsstellen und ihrer Umgebung sowie erstmals auch die Verknüpfung der Stoffgehalte in den Moosen mit denen der flächendeckend modellierten Gesamtdeposition von Blei. Letztere wurde durch das European Monitoring and Evaluation Programme (EMEP) in einer Auflösung von $50 \times 50 \mathrm{~km}^{2}$ zur Verfügung gestellt. EMEP ist ein wissenschaftlich fundiertes, internationales Programm, das im Rahmen der Convention on Long-range Transboundary Air Pollution (CLRTAP) großräumige grenzüberschreitende Luftverunreinigungen in Europa erfasst und modelliert.

Material und Methoden In Baden-Württemberg erfolgte die Bestimmung der Anreicherung von Metallen überwiegend in Hypnum cupressiforme seit 1990 und von Stickstoff 2005 nach europaweit harmonisierter Methodik qualitätskontrolliert. Die Daten der chemischen Analysen wurden mit den topografischen und ökologischen Beschreibungen der bis zu 78 Beprobungsorte und mit Informationen über die Landnutzung in ihrer Umgebung in dem WebGIS MossMet zusammengeführt und ausgewertet: Die räumliche Struktur der Metall- und Stickstoffanreicherung wurde per Variografie analysiert und modelliert sowie mit Kriging-Interpolation flächenhaft dargestellt. Aus den standort- und metallspezifischen Messdaten sowie den daraus geostatistisch berechneten Flächendaten über die Metallakkumulation wurde ein zusammenfassender Multimetallindex $\left(\mathrm{MMI}_{1990-2005}\right)$ jahresübergreifend für $\mathrm{As}, \mathrm{Cd}$, $\mathrm{Cr}, \mathrm{Cu}, \mathrm{Fe}, \mathrm{Ni}, \mathrm{Pb}, \mathrm{Ti}, \mathrm{V}$ und $\mathrm{Zn}$ berechnet und kartiert. Die Zusammenhänge zwischen den Schwermetallakkumulationen, Standortcharakteristika sowie Landnutzung und Emissionen wurden mit Rangkorrelationskoeffizienten und Kontingenzmaßen sowie am Beispiel von $\mathrm{Pb}$ multivariatstatistisch mit Chisquare Automatic Interaction Detection (CHAID) quantifiziert. Erstmals gelang es, die Stoffgehalte in den Moosen mit denen der europaweit flächendeckend 
modellierten EMEP-Gesamtdepositionsdaten am Beispiel von Blei zu verknüpfen und korrelationsstatistisch auszuwerten.

Ergebnisse Die Moosanalysen zeigen von 1990 bis 2005 einen statistisch signifikanten Rückgang der Bioakkumulation für die meisten Metalle. Nur die Cr- und Sb-Gehalte in den Moosen nehmen von 2000 bis 2005 zu, jedoch nicht statistisch signifikant. Auch der Rückgang des MMI zwischen 2000 und 2005 ist statistisch nicht signifikant. Die Stickstoffgehalte in den baden-württembergischen Moosen rangieren zwischen 1,15 und $1,74 \%$ und sind negativ mit der Bestandeshöhe korreliert $\left(r_{\mathrm{s}}=0,43, p<0,01\right)$. Die Rangkorrelationskoeffizienten, die den statistischen Zusammenhang zwischen Metallgehalten in den Moosen und die Landnutzung im Umkreis der Beprobungsorte kennzeichnen, rangieren zwischen $r_{\mathrm{s}}=0,3$ und $r_{\mathrm{s}}=0,7(p<0,05)$. Von den Standortmerkmalen weisen vor allem die Variablen Waldflächenanteil (insbesondere $\mathrm{Cd}, \mathrm{Pb}, \mathrm{Zn}$ ), Flächendichte agrarischer Landnutzung ( $\mathrm{Cd}, \mathrm{Hg}, \mathrm{Pb}, \mathrm{Zn})$, Niederschlagssumme im Akkumulationszeitraum ( $\mathrm{Cd}, \mathrm{Pb}, \mathrm{Zn})$, orografische Höhe ( $\mathrm{Cr}, \mathrm{Fe}, \mathrm{V})$, Bestandeshöhe (As, $\mathrm{Hg}, \mathrm{Pb}, \mathrm{V}$ ) und Entfernung der Moosentnahmestelle von Straßen (Hg), Baumkronen oder Sträuchern $(\mathrm{Cu}, \mathrm{Hg}, \mathrm{Pb}, \mathrm{V})$ signifikante Korrelationen zur Metallanreicherung auf. Ohne Berücksichtigung der EMEP-Depositionsdaten für Cd identifiziert die multivariat-statistische Analyse mit CHAID den Waldflächenanteil im Umkreis von $5 \mathrm{~km}$ um die Moossammelstelle sowie die dortige Geländeneigung und Höhenlage als wichtigste Einflussgrößen für die Cd-Gehalte in den Moosen 2005. Die Pb-Gesamtdeposition (EMEP) und die PbGehalte in Moosen Baden-Württembergs sind miteinander korreliert (2005: $r=0,52, p<0,01)$.

Diskussion Erstmals konnte in Baden Württemberg am Beispiel von $\mathrm{Pb}$ mit einer landesweit flächendeckenden GIS-gestützten Korrelationsanalyse gezeigt werden, dass die in den Moosen gemessenen Anreicherungen mit der modellierten Gesamtdeposition (EMEP) verknüpft sind. Die Stärke der statistischen Zusammenhänge erwies sich allerdings als zeitlich nicht stabil. Die hier gefundenen statistischen Beziehungen zwischen Depositionen und Stoffanreicherungen in Moosen müssen durch Vergleiche mit empirischen Messungen ergänzt werden. Erste diesbezügliche Pilotauswertungen erfolgten durch die räumliche Verknüpfung des bundesweiten Moosmonitoringnetzes mit Messnetzen, in denen die atmosphärische Deposition quantitativ bestimmt wird (z. B. dem ICP-Forests-Level-2-Messnetz). Durch das Moosmonitoring könnten die im Rahmen des ICP Mapping and Modelling erzeugten modellierten Depositionskarten validiert werden. Derartige Depositionskarten werden für die Berechnung praxisrelevant regional differenzierter Überschreitungen der Critical Loads benötigt. Ferner könnten mithilfe des Moosmonitorings die räumlich gering aufgelösten EMEP-Karten zu höher aufgelösten Depositi- onskarten verrechnet werden. Im Vergleich zu zeitlich höher aufgelösten Depositionsmessungen erfasst das Moosmonitoring großräumig ein breites Stoffspektrum, das auch in anderen Messnetzen selten gemessene Stoffe mit humantoxikologischer Bedeutung (z. B. As, Al, Hg, Sb, V) umfasst. Das standardisierte Biomonitoring von Luftverunreinigungen mit Bodenmoosen bildet also ein wichtiges Bindeglied zwischen der technischen Erfassung von Stoffeinträgen durch Deposition und der Anreicherung dieser Stoffe in biologischem Material.

Schlussfolgerungen Das Moosmonitoring liefert wesentliche Beiträge zum Schwermetall- und zum Multikomponentenprotokoll der Convention on Long-range Transboundary Air Pollution (CLRTAP). Es belegt flächendeckend, wie sich Luftreinhaltepolitik auf die Anreicherung von atmosphärischen Stoffeinträgen in Schutzgütern wie der Vegetation auswirkt. Von besonderer umweltpolitischer Bedeutung ist, dass in keinem anderen Messprogramm räumlich so verdichtet Daten über ein breites, ökotoxikologisch und humanmedizinisch bedeutsames Stoffspektrum erhoben werden. Die räumliche Auflösung von Umweltinformationen ist ein wesentliches Kriterium für ihre Nutzbarkeit im Vollzug umweltpolitischer Maßnahmen auf Bundes- und Länderebene.

Empfehlungen und Perspektiven Das Moosmonitoring sollte langfristig fortgesetzt werden, denn es ist ein konsequent organisiertes Programm der Umweltbeobachtung, das über drei räumliche Ebenen - Region (z. B. Bundesland oder Naturraum), Staat (z. B. Deutschland) und Kontinent (z.B. Europa) - methodisch abgestimmte, qualitätskontrollierte flächendeckende Daten zu Stoffanreicherungen in der belebten Umwelt über ein internetfähiges WebGISPortal bereitstellt. Zudem liefert das Moosmonitoring als einziges Messnetz in Europa räumlich hinreichend differenzierte, flächendeckende Informationen über die Stickstoffexposition naturnaher und agrarisch beeinflusster Ökosysteme, die auch für einzelne Staaten und deren administrative Untergliederungen wie etwa Bundesländer räumlich aussagekräftig sind. Die in anderen Untersuchungen jüngst belegten europaweiten Korrelationen zwischen Stoffanreicherungen in Moosen und EMEP-Depositionsdaten werden in weiteren Berechnungen dazu genutzt, die Kartierung der Metall- und Stickstoffdepositionen räumlich höher aufzulösen, um den Anforderungen aus Wissenschaft und Praxis besser als bislang entsprechen $\mathrm{zu}$ können.

Schlüsselwörter Bioakkumulation · CHAID (Chisquare Automatic Interaction Detection) - EMEP (European Monitoring and Evaluation Programme) $\cdot$ Pb-Deposition - Geostatistik · ICP Vegetation (International Cooperative Programme on Effects of Air Pollution on Natural Vegetation and Crops) $\cdot$ Schwermetalle $\cdot$ Stickstoff 


\section{Monitoring the bioaccumulation of metals and nitrogen as part of the long-term integrated environmental monitoring in Baden-Württemberg}

Abstract Background, aim, and scope Since 1990 the UN ECE Heavy Metals in Mosses Surveys provide data inventories of the atmospheric heavy metal bioaccumulation in mosses across Europe. In the 2005 survey the nitrogen accumulation was measured for the first time in most of the participating countries. In Germany, the surveys were conducted in close cooperation with the relevant authorities of both the Federal Republic and the sixteen states. Therefore, statistical evaluations of the moss survey data with regard to the whole German territory and single federal states are of interest. This article concentrates on the federal state BadenWürttemberg, dealing with the mapping of the spatiotemporal trends of metal accumulation from 1990 to 2005, the spatial patterns of nitrogen accumulation in 2005, and the spatial variability of bioaccumulation due to characteristics of the sampling sites and their surroundings. Furthermore, for the first time the relationship between the element concentrations in the mosses and Europe-wide modelled data on total depositions from the European Monitoring and Evaluation Programme (EMEP) on total depositions and were evaluated by means of correlation analysis using lead as an example. EMEP is a scientifically based programme under the Convention on Long-range Transboundary Air Pollution (CLRTAP) for international co-operation to model transboundary air pollution problems.

Materials and methods In Baden-Württemberg the bioaccumulation of metal elements was determined mainly in Hypnum cupressiforme moss samples since 1990 and in 2005 also nitrogen was investigated according to a Europewide harmonised methodology. The according experimental protocol describes the selection of sampling sites and moss species, the chemical analysis and quality control and the dissemiation of the measured values for mapping spatial patterns. In Baden-Württemberg all sampling sites were described with regard to topographical and ecological characteristics and several criteria to be fulfilled according to the guideline. Together with the measurements this metadata was combined with other information regarding land use in the surroundings of the sampling sites in the WebGIS MossMet. The spatial structure of the metal bioaccumulation was analysed and modelled by variogram analyses and then mapped by applying different kriging techniques. Furthermore, multi metal indices (MMI) were derived for both the sampling sites and raster maps with help of percentile statistics: The $\mathrm{MMI}_{1990-2005}$ was calculated for $\mathrm{As}, \mathrm{Cd}, \mathrm{Cr}$, $\mathrm{Cu}, \mathrm{Fe}, \mathrm{Ni}, \mathrm{Pb}, \mathrm{Ti}, \mathrm{V}$ and $\mathrm{Zn}$. The statistical association of the metal bioaccumulation, site specific characteristics as well as information on land use and emissions were analysed by bivariate nonparametric correlation analysis, con- tingency tables and Chisquare Automatic Interaction Detection (CHAID).

Results The moss analyses show a statistically significant decrease of the bioaccumulation of most metal elements from 1990 till 2005. Only the $\mathrm{Cr}$ and $\mathrm{Sb}$ concentrations in the mosses increased from 2000 till 2005, however not statistically significant. Also the decline of the MMI from 2000 till 2005 is not statistically significant. The nitrogen concentrations in the mosses sampled in Baden-Württemberg range from 1.15 to $1.74 \%$ and are negatively correlated with the tree height $\left(r_{\mathrm{s}}=0.43, p<0.01\right)$. The rank correlation coefficients which reflect the statistical association between the metal concentrations in the mosses and the land uses in the surroundings of the sampling sites range from $r_{\mathrm{s}}=0.3$ to $r_{\mathrm{s}}=0.7(p<0.05)$. Among the site descriptors mainly the variables proportion of forested land uses (especially $\mathrm{Cd}, \mathrm{Pb}, \mathrm{Zn}$ ), proportion of agricultural land uses $(\mathrm{Cd}, \mathrm{Hg}, \mathrm{Pb}, \mathrm{Zn})$ precipitation sum $(\mathrm{Cd}, \mathrm{Pb}, \mathrm{Zn}$ ), altitude (Cr, Fe, V), tree height (As, $\mathrm{Hg}, \mathrm{Pb}, \mathrm{V})$ and the distance of the sampling site to the nearest road $(\mathrm{Hg})$ as well as to tree crowns and bushes $(\mathrm{Cu}, \mathrm{Hg}, \mathrm{Pb}, \mathrm{V})$ feature significant correlations with the metal concentrations in the mosses. Without consideration of the EMEP deposition data the multivariate statistical CHAID analysis identifies the proportion of forested land uses in a radius of $5 \mathrm{~km}$ around the sampling site as well as the slope and altitude as the statistically most significant factors for the Cd concentrations in the mosses sampled in 2005. The total deposition of $\mathrm{Pb}$ (EMEP) and the $\mathrm{Pb}$ concentrations in mosses in Baden-Württemberg are correlated (2005: $r=0.52, p<0.01$ ).

Discussion For the first time it could be shown that element concentrations measured in mosses of Baden-Württemberg are associated with modelled total depositions of lead (EMEP). The strength of the statistical correlations was found to vary with time. The comparison of atmospheric depositions and element concentration in mosses should be repeated with measured deposition data. In a pilot study this was carried out with data coming from e. g. the ICP Forest Level 2 data on throughfall and open field bulk deposition measurements. By relating data on atmospheric depositions with those on element concentration in mosses modelled deposition maps from the ICP Mapping and Modelling Programme could be validated. Furthermore the rather low resolution of the EMEP maps could be enhanced. Highly resolved deposition data is needed for the calculation of practice-oriented regionally differentiated exceedances of critical loads. In comparison with the deposition measurements, which feature a higher temporal resolution, the moss monitoring spaciously encompasses a wide spectrum of elements containing also elements with a human-toxicological relevance (e.g. As, $\mathrm{Al}, \mathrm{Hg}, \mathrm{Sb}, \mathrm{V}$ ) which are rarely measured in other monitoring networks. Hence the standardised biomonitoring of air pollutions with ectohydric mosses forms an important link between the technical acquisition 
of element depositions and their accumulation in biological material.

Conclusions The moss surveys contribute to the heavy metal and the multi-component-model of the Convention on Long-range Transboundary Air Pollution (CLRTAP) because they show on different spatial scales how air pollution control influences the accumulation of emitted substances in environmental subjects of protection like vegetation. If environmental monitoring is seen as a continuous task and the applied methodology works well as an early warning system then environmental policy is enabled to act in preventative way and to pursue unexpected developments. No other environmental monitoring programme provides such a wide range of ecotoxicologically relevant elements measured as spatially dense as the case for the moss surveys. The spatial distribution of environmental information is an essential criterion for their usability in terms of political measures for the federal states and the federation.

Recommendations and perspectives The Heavy Metals in Mosses Surveys are a good example for environmental monitoring activities reaching across three spatial and administrative levels: regional (e.g. federal state or natural landscape), nation wide (e.g. Germany) and continental (e. g. Europe). In Germany the harmonised and quality controlled moss data are made available via a WebGIS portal. Therefore, the moss data may easily be accessed for environmental monitoring purposes and the control of environmental political actions. Hence, the continuous task of environmental monitoring can be met and carried on in the future. Further, the moss monitoring is the only monitoring network in Europe which provides sufficiently differentiated area-wide data on the nitrogen exposure of semi-natural and agriculturally influenced ecosystems, which are also spatially meaningful for single countries and their administrative subdivisions (e. g. federal states). The Europe-wide correlations between element concentrations in mosses and modelled EMEP deposition data proved in other recent studies will be used to improve the spatial resolution of metal and nitrogen deposition mapping in order to comply with the requirements of science and praxis better than so far.

Keywords Bioaccumulation - CHAID (Chisquare Automatic Interaction Detection) $\cdot$ EMEP (European Monitoring and Evaluation Programme) $\cdot \mathrm{Pb}$ deposition . Geostatistics $\cdot$ Heavy metals $\cdot$ ICP Vegetation (International Cooperative Programme on Effects of Air Pollution on Natural Vegetation and Crops) $\cdot$ Nitrogen

\section{Hintergrund, Ziel und Bereich}

Stabilität und Resilienz von Ökosystemen sind wichtige Voraussetzungen für deren langfristig ertragreiche Nutzung
(Gigon und Grimm 1997) und insofern Schlüsselgrößen nachhaltiger Entwicklung. Deshalb ist es folgerichtig, dass Umweltmonitoring Kernbestandteil der Nachhaltigkeitsstrategien des UNESCO-Programms Man and the Biosphere (MaB) und der UNECE ist. ${ }^{1}$ Um Umweltveränderungen und ihre Ursachen detailliert und zuverlässig aufzeigen zu können, bedarf es neben einer medienspezifisch ausgerichteten Umweltüberwachung auch einer medienübergreifenden Umweltbeobachtung. Das übergeordnete Ziel dieser Art des Umweltmonitorings ist die langfristige Erfassung der Veränderung sowie der Belastung und Belastbarkeit der Umwelt als Ganzes (Rüdel et al. 2007). Die Verknüpfung von biologischen, chemischen und physikalischen Daten aus einzelnen Messnetzen ist Grundlage einer integrativen, medienübergreifenden Datenauswertung (Broecker et al. 2007). Hierfür ist es wichtig, auf langjährig bewährte Messnetze zurückgreifen und über gezielte Auswertungen Ergebnisse für die Politikberatung und eine fundierte Risikokommunikation bereitstellen zu können. Dies erfolgt in Baden-Württemberg durch medienübergreifende Umweltbeobachtung in Verbindung mit einer Schwerpunktsetzung. Schwerpunkte sind Wirkungen von Klimaveränderungen und Anpassungen der belebten Umwelt, Toxizität und Anreicherung chemischer Stoffe in der Umwelt, Wirkungen des Einsatzes neuer Technologien auf die Umwelt sowie Analyse und Bewertung des Stoffhaushaltes ausgewählter Umweltschadstoffe (LfU 2003; Schröder et al. 2005, 2006; LUBW 2009).

Da Moose über mehrere Jahre die Anreicherung von Metallen ohne physiologische Beeinträchtigung tolerieren, sind sie für das Monitoring der Bioakkumulation atmosphärischer Depositionen geeignet - von der lokalen über die regionale bis hin zur kontinentalen Ebene (Bealey 2008 a,b; Forster et al. 1993; Genßler et al. 2001; Herpin et al. 2004; Kostka-Rick et al. 2001; Mohr 1999, 2007; Rühling und Tyler 1968, 1969, 1970; Schröder et al. 2008; Tyler 1990; Wappelhorst et al. 2000; Zechmeister et al. 2006). Deutschland beteiligt sich seit 1990 alle fünf Jahre an den Heavy Metals in Mosses Surveys, die von der UNECE im ICP Vegetation auf Grundlage des Genfer Luftreinhalteabkommens (Convention on Long-Range Transboundary Air Pollution, CLRTAP) im Fünfjahresturnus an mindestens 1,5 Standorten pro $1000 \mathrm{~km}^{2}$ europaweit durchgeführt werden. Dabei werden die Anreicherungen von Schwermetallen (seit 1990) und Stickstoff (seit 2005) ermittelt. Die Ergebnisse werden in Berichten der Working Group on Effects (WGE) der CLRTAP und des Programmzentrums des International Cooperative Programme on Effects of Air Pollution on Natural Vegetation and Crops (ICP Vegetation) in Bangor, Wales veröffentlicht (Harmens et al. 2008). Die aktuellste Auswertung der Metall- und Stickstoffgehalte in Deutschland zwischen 1990 und 2005 legten Schröder et al. (2009) vor.

\footnotetext{
${ }^{1}$ Siehe auch http://www.unece.org/env/welcome.html.
} 
In Deutschland führen Bund und Länder das Moosmonitoring gemeinsam durch: Im Auftrag des Umweltbundesamtes (UBA) erfolgt die chemische Analyse der Moose und die Datenauswertung, die Bundesländer übernehmen die Moosprobenentnahme. Der Arbeitskreis Bioindikation/ Wirkungsermittlung der Landesämter und -anstalten für Umweltschutz (AKB) und ein Vertreter des UBA begleiten das Moosmonitoring als wissenschaftlicher Beirat. Folglich besteht Interesse an bundesweiten und länderspezifischen Auswertungen. Dieser Artikel behandelt die Kartierung der Metallanreicherung in Baden-Württemberg seit 1990, der Stickstoffanreicherung seit 2005 sowie die räumliche Varianz der Metallbioakkumulation in Abhängigkeit von Eigenschaften der Probeentnahmestellen und ihrer Umgebung.

\section{Material und Methoden}

\subsection{Datenerhebung}

Das Moosmonitoring wurde in Deutschland 1990, 1995, 2000 und 2005 entsprechend der europaweit verwendeten Richtlinie (Harmens 2005) durchgeführt. Dabei entfielen auf die Fläche Baden-Württembergs $\left(35751 \mathrm{~km}^{2}\right) 59$ (1990), 69 (1995), 78 (2000) bzw. 56 Moossammelorte, was einer räumlichen Dichte von 1,7, 2,1, 2,2 bzw. 1,6 Probenorten pro $1000 \mathrm{~km}^{2}$ entspricht und damit die Mindestvorgabe der Richtlinie (1,5 Moosprobenentnahmestandorte pro $1000 \mathrm{~km}^{2}$ ) erfüllt. Das Messnetz wurde zwischen 2000 und 2005 ohne Einschränkung seiner geostatistischen Validität und Landschaftsrepräsentanz ausgedünnt (Pesch et al. 2008; Schröder et al. 1991, 2004; Schröder und Schmidt 2000).

Die Moosprobenentnahme erfolgte in Anlehnung an die maßgebliche Richtlinie (Harmens 2005). Die Massenkonzentrationen der Elemente Aluminium ( $\mathrm{Al}$ ), Barium (Ba), Calcium (Ca), Kupfer (Cu), Eisen (Fe), Kalium (K), Magnesium (Mg), Mangan (Mn), Natrium (Na), Strontium (Sr), Titan (Ti) und Zink (Zn) wurden in der Aufschlusslösung mit ICP-OES nach DIN EN ISO 11885 (E 22) und VDLUFA Methodenbuch 2.2.2.6 gemessen. Die Massenkonzentrationen der Elemente Arsen (As), Cadmium (Cd), Kobalt (Co), Chrom (Cr), Molybdän (Mo), Nickel (Ni), Blei $(\mathrm{Pb})$, Antimon $(\mathrm{Sb})$ und Vanadium $(\mathrm{V})$ wurden mit ICP-MS nach DIN 38406-29 (E 29) und VDLUFA Methodenbuch 2.2.2.5 bestimmt, die Gehalte von Quecksilber $(\mathrm{Hg})$ in den Moosen mit einem Quecksilberanalysator (thermostatisierte Zweiwegzelle) nach DIN EN 1483 und VDLUFA Methodenbuch VII. Die Massenkonzentration vom Gesamt-N (Gesamtstickstoff) wurde mit einem $\mathrm{C} / \mathrm{N}$-Analyser (Wärmeleitfähigkeitsdetektor) nach VDLUFA Methodenbuch II 3.5.2.7 durch die Verbrennung von 0,2 g Moosprobenmaterial im Sauerstoffstrom ermittelt. Bei dieser Methode wird
Stickoxid zu molekularem N reduziert und mit einem Wärmeleitfähigkeitsdetektor bestimmt.

Die Qualität der Messungen wurde in zwei Schritten kontrolliert: Sie erfolgte zunächst in den einzelnen Teilnehmerstaaten und umfasste die Messung der Referenzmaterialien M2 (hohe Metallkonzentrationen) und M3 (Hintergrundwerte) in jeder Messserie sowie den Austausch und die Messung von Moosproben, die auf beiden Seiten der Grenzen benachbarter Staaten gesammelt wurden. Anschließend wurden alle qualitätskontrollierten Daten der Teilnehmerstaaten vom Programmzentrum des ICP Vegetation (http://icpvegetation.ceh.ac.uk/) erneut daraufhin überprüft, ob sie den Anforderungen nach Steinnes et al. (1997) entsprechen. In den deutschen Moosmonitoringkampagnen wurde mit großer Sorgfalt vorgegangen - vom Messnetzdesign über die Schulung der Moosprobensammler, die Probenentnahme einschließlich Dokumentation, die chemische Analytik und Qualitätskontrolle bis zur Datenauswertung (Schröder et al. 1991, 2004). Zur Sicherung und Kontrolle der analytischen Qualität wurden alle üblichen Maßnahmen ergriffen (Funk et al. 2006) und ausführlich dargestellt (Schröder et al. 2009). Bevor die qualitätskontrollierten Daten von den Teilnehmerstaaten an das Programmzentrum zur Plausibilitätsprüfung aller europaweit gemessenen Daten geschickt wurden, erfolgte eine Plausibilitätsprüfung in jedem Teilnehmerstaat und gegebenenfalls der Ausschluss einzelner Datensätze von der Auswertung. In Deutschland erfolgt die Durchführung von Plausibilitätsuntersuchungen durch die Bundesländer. Am Beispiel der Messkampagne 2005 wurden je ein kompletter Standortdatensatz aus Bayern und Sachsen-Anhalt sowie zwölf Messwerte von zehn nordrhein-westfälischen Standorten und zwei Messwerte von zwei bayrischen Standorten als nicht plausibel eingestuft (Pesch et al. 2007b). In Baden-Württemberg wurden 2005 hingegen alle gemessenen Werte als plausibel eingestuft. Die Überprüfung erfolgte sowohl anhand statistischer Kriterien (Messwerte oberhalb des 98. Perzentils) wie auch der fachlichen Expertise der zuständigen Sachbearbeiter der Bundesländer.

\subsection{Datenauswertung}

Zur Kartierung der räumlichen Muster der Bioakkumulation einzelner Metallelemente oder des metalleübergreifenden Multimetallindex (MMI, s. u.) wurden geostatistische Methoden eingesetzt (Matheron 1965; Webster und Oliver 2001). Die Qualität der dazu berechneten Flächenschätzungen wurde durch Kreuzvalidierung ermittelt (Johnston et al. 2001; Pesch 2003, Pesch et al. 2007b). Der MMI wurde sowohl für die Messwerte als auch für die aus ihnen berechneten flächenhaften Schätzwerte ermittelt: Dazu wurden pro Element 10 Perzentilklassen gebildet, und jedem Perzentil wurde dann ein Indexwert zwischen 1 (niedrige Ak- 
kumulation) und 10 (hohe Anreicherung) zugewiesen. Der $\mathrm{MMI}_{1990-2005}$ eines jeden Moossammelortes oder einer jeden Rasterzelle entspricht dem Durchschnitt der dort ermittelten elementspezifischen Indexwerte der Konzentrationen von $\mathrm{As}, \mathrm{Cd}, \mathrm{Cr}, \mathrm{Cu}, \mathrm{Fe}, \mathrm{Ni}, \mathrm{Pb}, \mathrm{V}$, Ti und $\mathrm{Zn}$, die durchgängig 1990, 1995, 2000 und 2005 in Baden-Württemberg gemessen bzw. geostatistisch geschätzt wurden.

Alle Ergebnisse und weiterführende Informationen der Moosmonitoringkampagnen wurden im WebGIS MossMet dokumentiert und sind Bund, Ländern sowie dem ICP Vegetation zugänglich (Kleppin et al. 2008; Pesch et al. 2007a). Die elementspezifischen Messdaten der Monitoringkampagnen 1990, 1995, 2000 und 2005 sowie die daraus berechneten MMI konnten daher im Hinblick auf folgende Informationen korrelationsstatistisch untersucht werden: 1) standortspezifische Informationen (s.u.) im Sinne der Verfahrensrichtlinie (Harmens 2005), ökologische und topografische Standorteigenschaften, Ablauf und meteorologische Randbedingungen der Moosprobenentnahme, 2) flächenhafte Informationen über das Klima, die Höhenlage über NN, Flächenanteile forstlicher, agrarischer und urbaner Landnutzung nach CORINE Land Cover (Keil et al. 2005), Entfernung zu großen Industriebetrieben sowie Depositionen in der Umgebung der Moosmonitoringstandorte. Hierbei handelt es sich um Daten über atmosphärische Depositionen aus dem EMEP-Luftmessnetz des Umweltbundesamtes (wet only), aus dem ICP Forest-Messnetz (Freiland- und Bestandsdeposition) sowie Flächenschätzungen der modellierten Nass-, Trocken- und Gesamtdeposition.

Stärke und Richtung der statistischen Zusammenhänge zwischen a) jeweils zwei Metallgehalten in den Moosen, b) zwischen Metallkonzentrationen in Moosen und Depositionen (Wet only Freiland Messungen aus dem UBA Luftmessnetz und dem ICP Integrated Monitoring; Bulk Freiland und Bestandesmessungen aus dem ICP Forests Level 2 Messnetz) und c) zwischen Metallgehalten in Moosen und modellierter Nass-, Trocken- und Gesamtdeposition wurden durch Rangkorrelationskoeffizienten nach Spearman $r_{\mathrm{s}}$ quantifiziert. Zusätzlich wurden multivariat-statistische Korrelationsmuster mit Chi-square Automatic Interaction Detection (CHAID, Kass 1980) berechnet. Dabei bildeten die metrisch skalierten Gehalte der in den Moosen 2005 gemessenen Stoffgehalte jeweils die Zielvariable. Als Prädiktoren dienten die standortbeschreibenden Metainformationen sowie flächenhaft vorliegende Daten zur Umgebungsbeschreibung.

Um die Stickstoffgehalte in den Moosen um den Traufeeffekt zu bereinigen, erfolgte eine Umrechnung der Stickstoffakkumulationen mit den Schwefelgehalten in den Moosen. Dieses Vorgehen ist dadurch begründet, dass die S-Konzentrationen in Moosen bei aktuell sehr niedrigen $\mathrm{SO}_{2}-$ Immissionen großräumig nur gering variieren (Mohr 1999).
Unterschiede der S-Gehalte sind somit in großen Teilen Deutschlands vorrangig auf abweichende Depositionsbedingungen am Probenentnahmeort zurückzuführen. Aufgrund der hohen Korrelation sedimentierender S- und N-Einträge im Niederschlag (Dämmgen 2005) sowie solcher in den untersuchten Moospflanzen (2005: $r=0,83 ; p<0,0001$ ) kann der S-Gehalt als Indikator für den Traufeeinfluss der Begleitvegetation (vornehmlich Bäume und Sträucher) und zur Standardisierung der N-Gehalte herangezogen werden. Die Umrechnung basiert auf der folgenden empirischen Formel: $\mathrm{N}_{\text {korrigiert }}=[1000 / \mathrm{S}-$ Konzentration $] \times \mathrm{N}_{\text {gemessen }}$. Hierbei entspricht $1000 \mu \mathrm{g} / \mathrm{g} \mathrm{S}$ der zu erwartenden (und häufig festgestellten) S-Bioakkumulation unter Freilandbedingungen (außerhalb des Kronentraufeeinflusses).

\section{Ergebnisse}

Von 1990 bis 2005 ist Hypnum cupressiforme das am häufigsten in Baden-Württemberg gesammelte Moos, gefolgt von Pleurozium schreberi (maximal 11,9\% 1990), Scleropodium purum (maximal 3,4\% 1990) und Hylocomium splendens (1,7\% 1990) (Tab. 1). Hypnum cupressiforme wurde dabei fast ausschließlich von Baumstümpfen entnommen. Nur im Moosmonitoring 1995 wurde in zwei dokumentierten Fällen diese Moosart vom Boden gesammelt. Abbildung 1 zeigt die räumliche Verteilung der in BadenWürttemberg 1990, 1995, 2000 und 2005 beprobten Orte und Moosarten.

Tabelle 2 gibt anhand der Perzentilstatistik der 12 Standardmetalle und der zusammenfassenden MMI einen Überblick über die zeitliche Entwicklung der Bioakkumulation. Anhand des 20., 50. und 90. Perzentils sowie des MMI lässt sich eine deutliche, zumeist kontinuierliche Abnahme der Metallanreicherung in Moosen zwischen 1990 und 2005 nachweisen. Ausnahmen bilden $\mathrm{Cr}$ und Sb, die beide 2005 gegenüber 2000 angestiegen sind - allerdings nicht signifikant (Tab. 3). Der MMI nahm von 2000 bis 2005 ab, aber nicht signifikant (Tab. 3).

Die räumliche Differenzierung der in Tab. 2 zusammenfassend beschriebenen zeitlichen Entwicklung der Stoffanreicherung wird am Beispiel des $\mathrm{MMI}_{1990-2005}$ in Abb. 2

Tabelle 1 Beprobte Moosarten in Baden-Württemberg 1990 bis 2005

\begin{tabular}{ccccccccc}
\hline & $\begin{array}{c}\text { Hypnum } \\
\text { cupressiforme }\end{array}$ & \multicolumn{2}{c}{$\begin{array}{c}\text { Hylocomium } \\
\text { splendens }\end{array}$} & \multicolumn{2}{c}{$\begin{array}{c}\text { Pleurozium } \\
\text { schreberi }\end{array}$} & \multicolumn{2}{c}{$\begin{array}{c}\text { Scleropodium } \\
\text { purum }\end{array}$} \\
\hline & $n$ & $\mathbf{\%}$ & $n$ & $\mathbf{\%}$ & $n$ & $\mathbf{\%}$ & $n$ & $\mathbf{\%}$ \\
\hline 1990 & 49 & 83,1 & 1 & 1,7 & 7 & 11,9 & 2 & 3,4 \\
1995 & 64 & 92,8 & 0 & 0,0 & 4 & 5,8 & 1 & 1,4 \\
2000 & 76 & 97,4 & 0 & 0,0 & 2 & 2,6 & 0 & 0,0 \\
2005 & 54 & 96,4 & 0 & 0,0 & 2 & 3,6 & 0 & 0,0 \\
\hline
\end{tabular}


Abb. 1 Messnetzverteilung und beprobte Moosarten in Baden-Württemberg 1990-2005

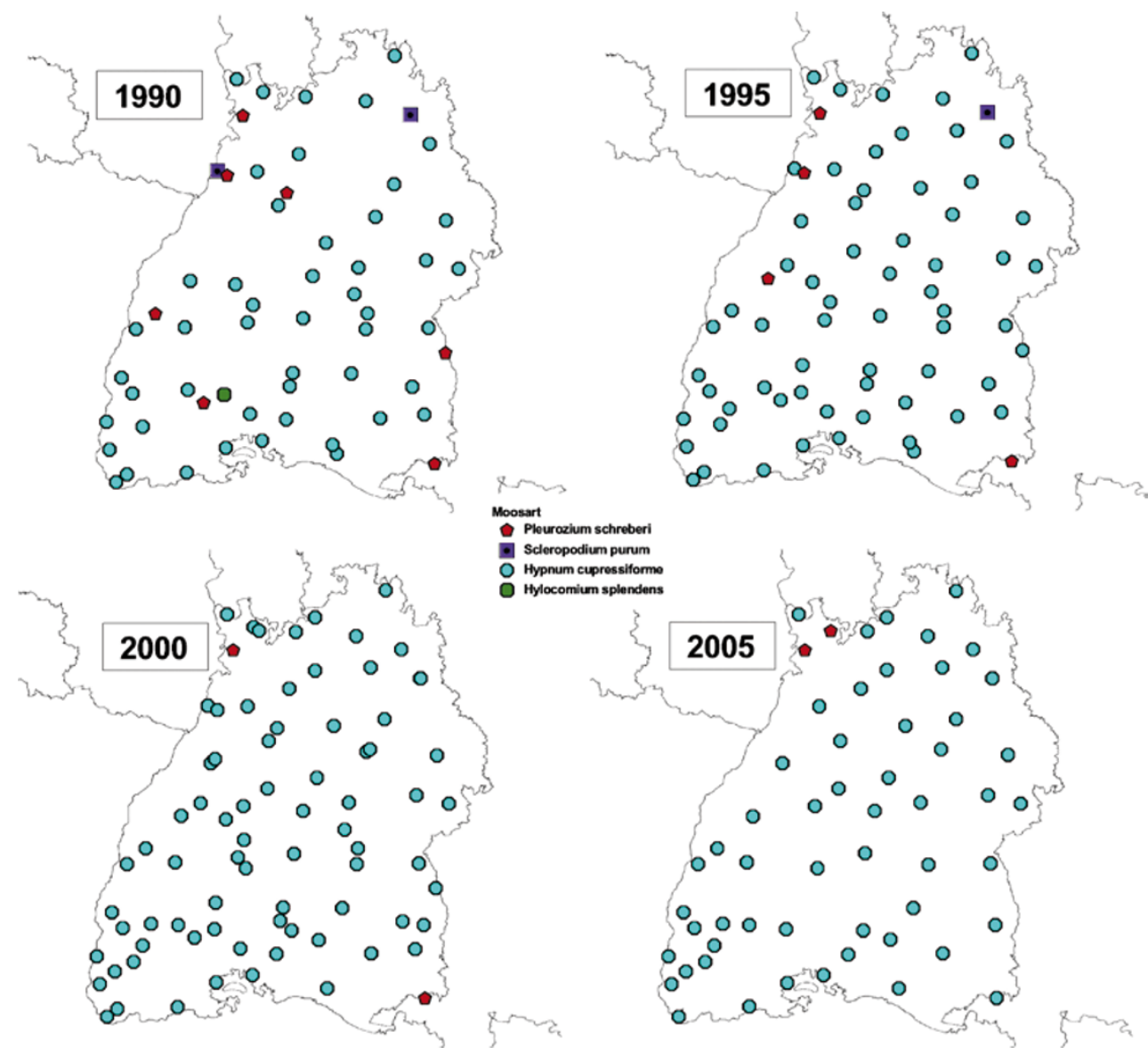

kartografisch veranschaulicht. Die Unschärfen der Flächenschätzung führen zu Abweichungen der auf Schätzungen basierenden MMI (Abb. 2) im Vergleich zu den MMI, die auf den exakten Messwerten beruhen (Tab. 3). Das räumliche Muster der Stickstoffanreicherung wird anhand der Originalmessdaten sowie den mit der Schwefelformel (Abschn. 2.2) umgerechneten Werten dargestellt (Abb. 3). Im zeitlichen Verlauf wird eine Reduktion der Anreicherung von Metallen und Schwermetallen in terrestrischen Ökosystemen Baden-Württembergs deutlich. Noch 1990 und 1995 zeigten weite Landesteile eine höhere Metall- und Schwermetallbelastung als bei den Untersuchungskampagnen der Jahre 2000 und 2005. Als belastete Regionen können für das Jahr 2005 der Südschwarzwald und der Odenwald genannt werden, die als typische Depositionsgebiete in der Westwinddrift eine Beaufschlagung mit luftgetragenen Schadstoffen, insbesondere Metallen und Schwermetallen, erfahren.

Auch hinsichtlich der Stickstoffakkumulation fallen der Südschwarzwald und der Odenwald durch höhere Stickstoffwerte auf, während die anderen Landesteile durch eine geringere Akkumulation gekennzeichnet sind.

Bei der Untersuchung von statistischen Zusammenhängen zwischen der Metallanreicherung in Moosen und
Eigenschaften der Moossammelorte und ihrer Umgebung wurden EMEP-Depositionsdaten mit einer räumlichen Auflösung von $50 \mathrm{~km} \times 50 \mathrm{~km}$ berücksichtigt. Abbildung 4 zeigt im linken Teil die räumliche Differenzierung der Pb-Anreicherungen in den Moosen, wobei die Mediane der Pb-Werte für jede EMEP-Rasterzelle anhand der Einzelmesswerte der in ihr gelegenen Beprobungspunkte berechnet wurden. Im rechten Abbildungsteil ist in derselben räumlichen Auflösung die $\mathrm{Pb}$-Gesamtdeposition dargestellt. Zwischen beiden räumlichen Verteilungen bestehen positive Rangkorrelationen $\left(r_{\mathrm{s}}\right)$ mit einem Maximalwert von $r_{\mathrm{s}}=0,52$ bei $p<0,01$.

In Ergänzung zu den bei der Moosprobenentnahme erhobenen Informationen über Eigenschaften der Sammelorte wurden flächenhafte Daten berücksichtigt, welche die Landnutzung und insofern potenzielle Flächenquellen für Metalle und Stickstoff in ihrer Umgebung kennzeichnen. Die Ergebnisse der bivariaten Zusammenhangsanalysen werden für metrische Daten in Tab. 4 in Form des Rangkorrelationskoeffizienten nach Spearman $\left(r_{\mathrm{s}}\right)$ präsentiert. Die Werte dieses Zusammenhangsmaßes liegen zwischen 0,3 und 0,7. Negativ korreliert sind die Stoffgehalte in Moosen mit der Nähe zu Bäumen und Sträuchern, die Stoffe aus der Luft filtern, die anschließend vom Nieder- 
Tabelle 2 Deskriptiv-statistische Maßzahlen der Elementgehalte in baden-württembergischen Moosen 1990-2005 (Perzentilangaben für die Elemente in $\mu \mathrm{g} / \mathrm{g}$ )
Tabelle 3 Inferenz-statistische Analyse der Entwicklung der Stoffakkumulationen in baden-württembergischen Moosen 1990-2005

\begin{tabular}{|c|c|c|c|c|c|c|c|c|c|c|c|c|c|}
\hline \multicolumn{14}{|c|}{1990} \\
\hline & As & Cd & $\mathrm{Cr}$ & $\mathrm{Cu}$ & $\mathrm{Fe}$ & Hg & $\mathbf{N i}$ & $\mathbf{P b}$ & Sb & Ti & $\mathbf{V}$ & $\mathbf{Z n}$ & MMI \\
\hline Anzahl & 59 & 59 & 59 & 59 & 59 & - & 58 & 59 & - & 59 & 59 & 59 & 59 \\
\hline Min & 0,156 & 0,189 & 1,23 & 4,54 & 279 & - & 1,09 & 9,08 & - & 16,1 & 1,45 & 25,2 & 4,8 \\
\hline Median & 5,370 & 0,645 & 4,27 & 21,10 & 1977 & - & 7,64 & 51,00 & - & 39,3 & 10,24 & 120,8 & 7,8 \\
\hline Max & 0,396 & 0,339 & 2,09 & 9,38 & 560 & - & 2,97 & 14,40 & & 22,6 & 2,79 & 45,0 & 9,9 \\
\hline \multicolumn{14}{|c|}{1995} \\
\hline & As & Cd & $\mathrm{Cr}$ & $\mathbf{C u}$ & $\mathrm{Fe}$ & $\mathbf{H g}$ & $\mathbf{N i}$ & $\mathbf{P b}$ & Sb & $\mathbf{T i}$ & $\mathbf{V}$ & Zn & MMI \\
\hline Anzahl & 69 & 69 & 69 & 69 & 69 & 69 & 69 & 69 & 69 & 69 & 69 & 69 & 69 \\
\hline Min & 0,051 & 0,144 & 0,66 & 4,35 & 170 & 0,001 & 0,66 & 3,41 & 0,081 & 8,1 & 0,58 & 21,8 & 2,3 \\
\hline Median & 2,690 & 1,450 & 7,64 & 16,10 & 1900 & 0,128 & 6,78 & 48,00 & 3,110 & 92,4 & 7,33 & 100,9 & 6,4 \\
\hline Max & 0,268 & 0,336 & 1,74 & 8,39 & 456 & 0,045 & 2,43 & 9,29 & 0,174 & 19,6 & 1,87 & 41,0 & 9,6 \\
\hline \multicolumn{14}{|c|}{2000} \\
\hline & As & Cd & $\mathrm{Cr}$ & $\mathrm{Cu}$ & $\mathrm{Fe}$ & $\mathrm{Hg}$ & $\mathbf{N i}$ & $\mathbf{P b}$ & $\mathbf{S b}$ & Ti & $\mathbf{V}$ & $\mathbf{Z n}$ & MMI \\
\hline Anzahl & 78 & 78 & 78 & 78 & 78 & 78 & 78 & 78 & 78 & 78 & 78 & 78 & 78 \\
\hline Min & 0,070 & 0,080 & 0,51 & 2,92 & 192 & 0,026 & 0,82 & 2,32 & 0,080 & 3,6 & 0,55 & 18,5 & 1,3 \\
\hline Median & 1,040 & 1,120 & 4,57 & 13,38 & 1561 & 0,145 & 4,33 & 21,30 & 0,500 & 49,9 & 3,88 & 82,4 & 4,4 \\
\hline Max & 0,195 & 0,230 & 1,10 & 7,08 & 460 & 0,052 & 2,04 & 5,25 & 0,165 & 8,8 & 1,41 & 34,5 & 9,4 \\
\hline \multicolumn{14}{|c|}{2005} \\
\hline & As & Cd & $\mathrm{Cr}$ & $\mathbf{C u}$ & $\mathrm{Fe}$ & Hg & $\mathbf{N i}$ & $\mathbf{P b}$ & Sb & Ti & V & $\mathrm{Zn}$ & MMI \\
\hline Anzahl & 56 & 56 & 56 & 56 & 56 & 56 & 56 & 56 & 56 & 56 & 56 & 56 & 56 \\
\hline Min & 0,050 & 0,070 & 0,79 & 4,08 & 113 & 0,018 & 0,53 & 1,44 & 0,090 & 1,2 & 0,34 & 18,2 & 1,3 \\
\hline Median & 0,610 & 0,840 & 5,55 & 14,06 & 1455 & 0,093 & 3,47 & 16,83 & 0,595 & 44,7 & 3,22 & 83,7 & 3,5 \\
\hline Max & 0,165 & 0,195 & 1,78 & 6,35 & 329 & 0,041 & 1,71 & 3,97 & 0,170 & 5,9 & 0,97 & 33,9 & 8,6 \\
\hline
\end{tabular}

\begin{tabular}{llllllllllllll}
\hline & As & $\mathbf{C d}$ & $\mathbf{C r}$ & $\mathbf{C u}$ & $\mathbf{F e}$ & $\mathbf{H g}$ & $\mathbf{N i}$ & $\mathbf{P b}$ & $\mathbf{S b}$ & $\mathbf{T i}$ & $\mathbf{V}$ & $\mathbf{Z n}$ & $\mathbf{M M I}$ \\
\hline $1990-1995$ & $\mathrm{~A}^{\mathrm{a}}$ & $\mathrm{A}^{\mathrm{c}}$ & $\mathrm{A}^{\mathrm{b}}$ & $\mathrm{A}^{\mathrm{b}}$ & $\mathrm{A}^{\mathrm{a}}$ & - & $\mathrm{A}^{\mathrm{b}}$ & $\mathrm{A}^{\mathrm{a}}$ & - & $\mathrm{A}^{\mathrm{a}}$ & $\mathrm{A}^{\mathrm{a}}$ & $\mathrm{A}^{\mathrm{b}}$ & $\mathrm{A}^{\mathrm{a}}$ \\
$1990-2000$ & $\mathrm{~A}^{\mathrm{a}}$ & $\mathrm{A}^{\mathrm{a}}$ & $\mathrm{A}^{\mathrm{a}}$ & $\mathrm{A}^{\mathrm{a}}$ & $\mathrm{A}^{\mathrm{a}}$ & - & $\mathrm{A}^{\mathrm{a}}$ & $\mathrm{A}^{\mathrm{a}}$ & - & $\mathrm{A}^{\mathrm{a}}$ & $\mathrm{A}^{\mathrm{a}}$ & $\mathrm{A}^{\mathrm{a}}$ & $\mathrm{A}^{\mathrm{a}}$ \\
$1990-2005$ & $\mathrm{~A}^{\mathrm{a}}$ & $\mathrm{A}^{\mathrm{a}}$ & $\mathrm{A}^{\mathrm{c}}$ & $\mathrm{A}^{\mathrm{a}}$ & $\mathrm{A}^{\mathrm{a}}$ & - & $\mathrm{A}^{\mathrm{a}}$ & $\mathrm{A}^{\mathrm{a}}$ & - & $\mathrm{A}^{\mathrm{a}}$ & $\mathrm{A}^{\mathrm{a}}$ & $\mathrm{A}^{\mathrm{a}}$ & $\mathrm{A}^{\mathrm{a}}$ \\
$1995-2000$ & $\mathrm{~A}^{\mathrm{b}}$ & $\mathrm{A}^{\mathrm{a}}$ & $\mathrm{A}^{\mathrm{a}}$ & $\mathrm{A}^{\mathrm{a}}$ & $\mathrm{Z}$ & $\mathrm{Z}$ & $\mathrm{A}^{\mathrm{b}}$ & $\mathrm{A}^{\mathrm{a}}$ & $\mathrm{A}^{\mathrm{c}}$ & $\mathrm{A}^{\mathrm{a}}$ & $\mathrm{A}^{\mathrm{a}}$ & $\mathrm{A}^{\mathrm{a}}$ & $\mathrm{A}^{\mathrm{a}}$ \\
$1995-2005$ & $\mathrm{~A}^{\mathrm{a}}$ & $\mathrm{A}^{\mathrm{a}}$ & $\mathrm{Z}$ & $\mathrm{A}^{\mathrm{a}}$ & $\mathrm{A}^{\mathrm{a}}$ & $\mathrm{A}^{\mathrm{a}}$ & $\mathrm{A}^{\mathrm{a}}$ & $\mathrm{A}^{\mathrm{a}}$ & $\mathrm{A}^{\mathrm{c}}$ & $\mathrm{A}^{\mathrm{a}}$ & $\mathrm{A}^{\mathrm{a}}$ & $\mathrm{A}^{\mathrm{a}}$ & $\mathrm{A}^{\mathrm{a}}$ \\
$2000-2005$ & $\mathrm{~A}^{\mathrm{a}}$ & $\mathrm{A}^{\mathrm{a}}$ & $\mathrm{Z}$ & $\mathrm{A}^{\mathrm{a}}$ & $\mathrm{A}^{\mathrm{a}}$ & $\mathrm{A}^{\mathrm{a}}$ & $\mathrm{A}^{\mathrm{a}}$ & $\mathrm{A}^{\mathrm{a}}$ & $\mathrm{Z}$ & $\mathrm{A}^{\mathrm{a}}$ & $\mathrm{A}^{\mathrm{a}}$ & $\mathrm{A}^{\mathrm{a}}$ & $\mathrm{A}^{\mathrm{c}}$ \\
\hline
\end{tabular}

schlag auf darunter liegende Moospolster befördert werden. Folglich besteht ein deutlicher Zusammenhang zwischen den $\mathrm{Cu}-G$ Gehalten in Moosen und der Entfernung zu Sträuchern $\left(r_{\mathrm{s}}=-0,7\right)$ sowie zu Bäumen $\left(r_{\mathrm{s}}=-0,5\right)$. Dieser Traufeffekt kommt bei allen Metallen außer bei As und Ni zur Ausprägung. Positiv korreliert sind die Konzentrationen von $\mathrm{As}, \mathrm{Cd}, \mathrm{Hg}, \mathrm{Pb}, \mathrm{V}$ und $\mathrm{Zn}$ mit den Niederschlägen. Je geringer der Anteil agrarisch genutzter Flächen im Umkreis der Beprobungsstellen, desto höher sind die Gehalte von $\mathrm{As}, \mathrm{Cd}, \mathrm{Cu}, \mathrm{Hg}, \mathrm{Ni}, \mathrm{Pb}, \mathrm{Sb}, \mathrm{V}$ und $\mathrm{Zn}$. Dieselbe Tendenz zeigen die Cd-Gehalte in Bezug auf den Anteil städtischer Bebauung und Verkehrsflächen im Umkreis der Moosbeprobungsstellen.

Multivariat-statistische Zusammenhänge der Cd-Anreicherung mit lokalen und regionalen Raummerkmalen werden in Abb. 5 am Beispiel von Cd (2005) aufgezeigt. Der Knoten 0 beschreibt die Verteilung der Cd-Messwerte an den 56 in Baden-Württemberg 2005 beprobten Standorten. Der Mittelwert der Untersuchungsstichprobe beträgt $0,20 \mu \mathrm{g} \mathrm{Cd} / \mathrm{g}$ Trockensubstanz Moos. Der Waldflächenanteil im Umkreis von $5 \mathrm{~km}$ um die Moossammelorte ist von den in Tab. 4 gelisteten Faktoren derjenige, der mit den Cd-Konzentrationen in den Moosen am engsten verknüpft ist: Je höher der Waldflächenanteil, desto höher sind die CdKonzentrationen in den gesammelten Hypnum-cupressiforme-Proben. Ebenfalls positiv korreliert sind Cd-Gehalte mit der Höhe der beprobten Orte über NN in Regionen mit einem Waldflächenanteil $>45,3 \%$. Die Geländeneigung ist mit den Cd-Gehalten auf Standorten mit Waldflächenanteilen $<28,7$ negativ verknüpft. 



Abb. 2 Zeitlicher Verlauf der Akkumulation von Metallen in Moosen in Baden-Württemberg 1990-2005

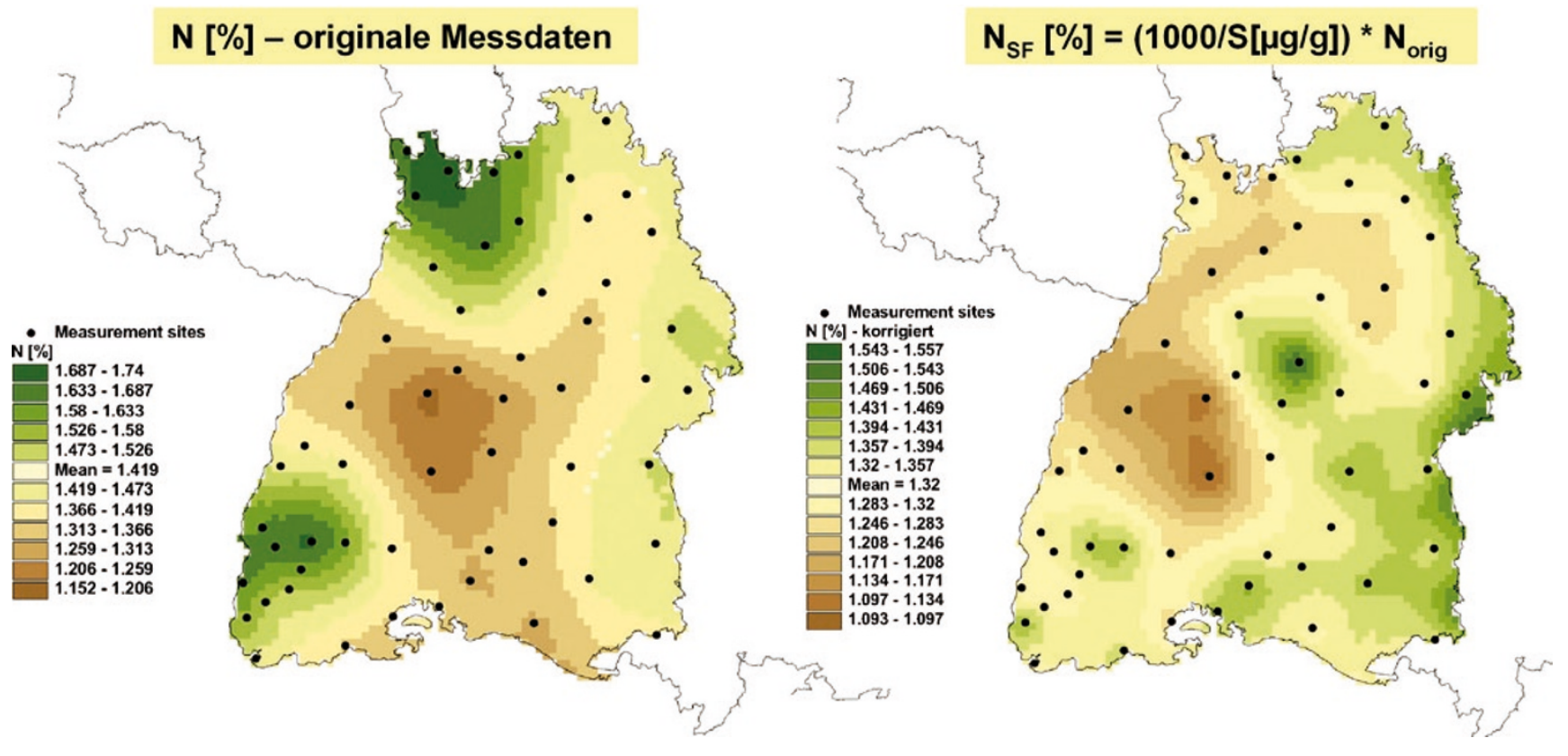

Abb. 3 Stickstoffakkumulation in Moosen in Baden-Württemberg 2005: originale Messdaten und am Schwefelgehalt der Moose standardisierte Stickstoffgehalte $\left(\mathrm{N}_{\mathrm{SF}}\right.$ : umgerechneter Stickstoffgehalt; $\mathrm{N}_{\text {orig }}$ : originaler Stickstoffgehalt) 



\section{EMEP - modellierte Deposition}

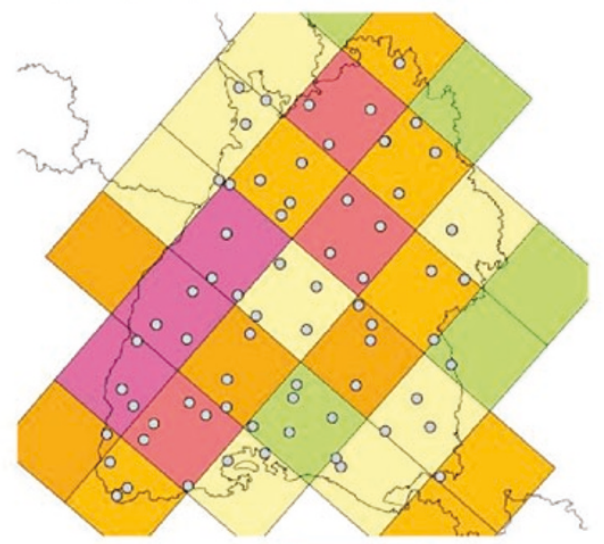

\section{5}

$r=0,31$

$(p<0,1)$
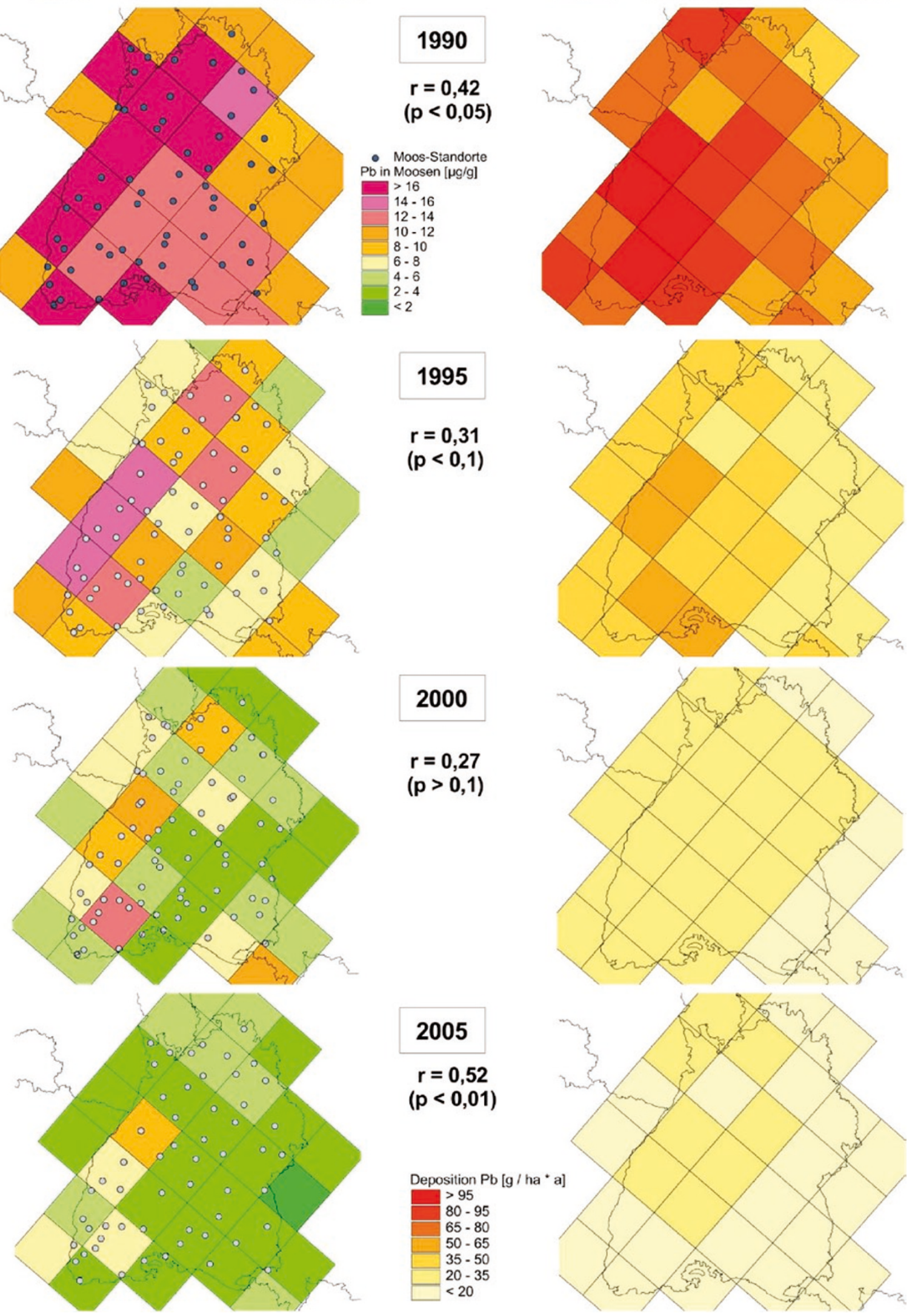

Abb. 4 Vergleich Pb in Moosen und modellierte Depositionen nach EMEP 1990 bis 2005 
Tabelle 4 Korrelationsanalytische Untersuchung von Einflussfaktoren auf die Stoffakkumulation in baden-württembergischen Moosen 1990-2005
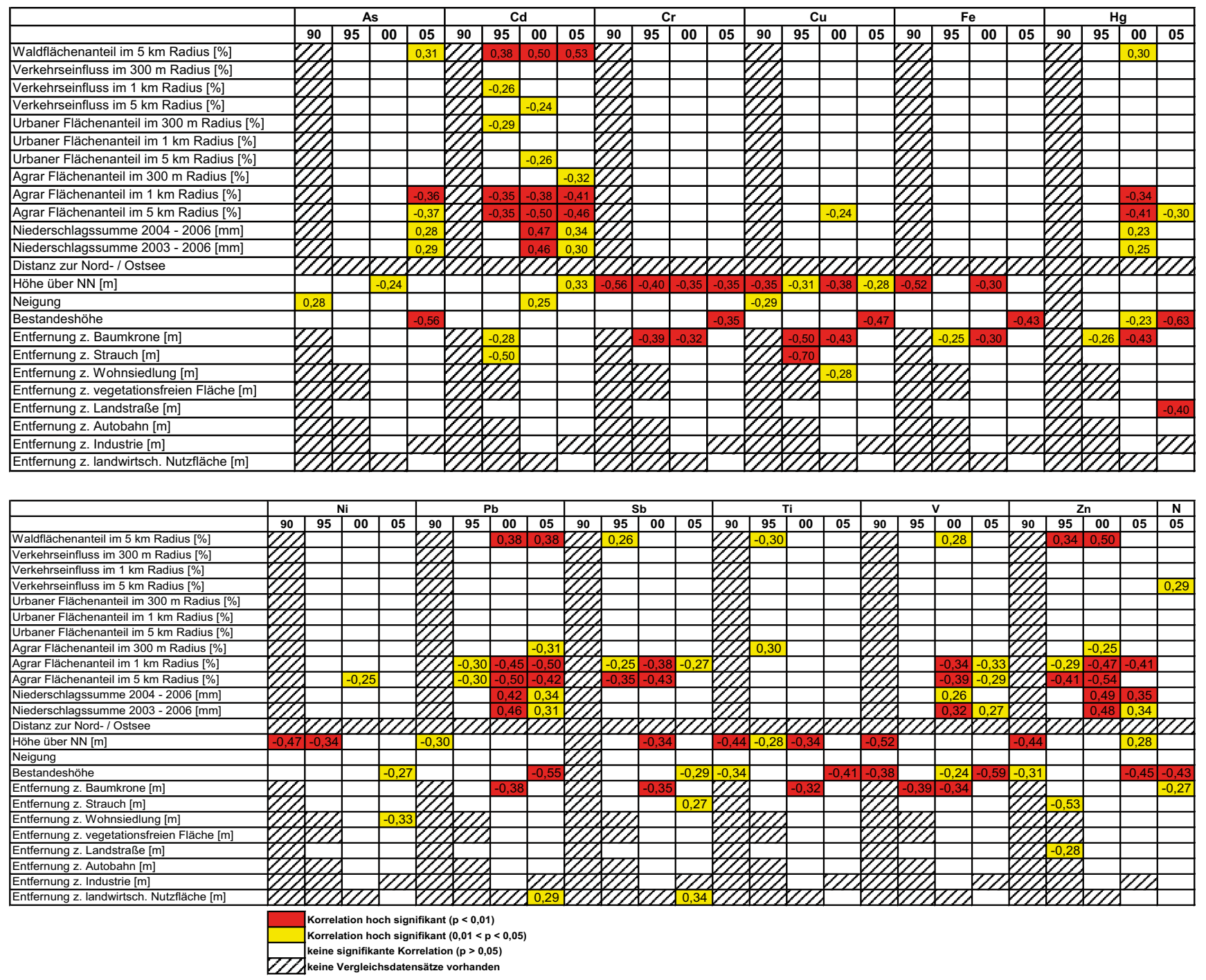

\section{Diskussion}

Die Ergebnisse der multivariat-statistischen Analyse mit CHAID bestätigen die Ergebnisse der bivariat-statistischen Berechnungen, ergänzen diese jedoch um Faktorenkombinationen. In diesen Kombinationen können sich dann auch solche Faktoren als bedeutsam erweisen, auf die die bivariate Analyse keine Hinweise lieferte - bei den Cd-Konzentrationen ist dies der Einfluss der Geländeneigung in Kombination mit dem Waldflächenanteil.

Auffällig an den baden-württembergischen Ergebnissen ist, dass es im Gegensatz zum Bundesmittel keine signifikante Erhöhung der Metallgehalte zwischen 2000 und 2005 gegeben hat. Dies trifft selbst auf die in der Westwinddrift gelegenen typischen Depositionsgebieten des Südschwarzwaldes und des Odenwaldes zu. Zwar sind $\mathrm{Cr}$ und $\mathrm{Sb}$ angestiegen, jedoch nicht signifikant.
Cr zeigte im Vergleich dazu im bundesweiten Trend die deutlichsten Anstiege zwischen 2000 und 2005. So ergab sich auf Bundesebene für den Zeitraum 2000-2005 eine signifikante Zunahme von 159 \% (Schröder et al. 2009). Die im Moos-Monitoring 2005 durchgeführten statistischen Auswertungen ergaben hoch signifikante negative Korrelationen der Cr-Gehalte mit den Niederschlagssummen innerhalb des Akkumulationszeitraums (Pesch et al. 2007b). Die Cr-Werte könnten somit auf den Einfluss von anhaftendem Bodenpartikel oder Feinstaubteilchen zurückzuführen sein, eine eindeutige Klärung dieses Sachverhalts konnte allerdings bislang nicht herbeigeführt werden. Folglich wurde empfohlen, mögliche, bis dato noch nicht berücksichtigte potenzielle Ursachen in die Auswertung zu integrieren. Hierzu zählen z.B. der Einfluss bislang wenig beachteter Immissionsquellen (z. B. Hausbrand) oder andere, direkt mit der Bodennutzung in 
Abb. 5 CHAID Baum-CdAkkumulation in Moosen in Baden-Württemberg 2005

\section{Cd $[\mu \mathrm{g} / \mathrm{g}]$}

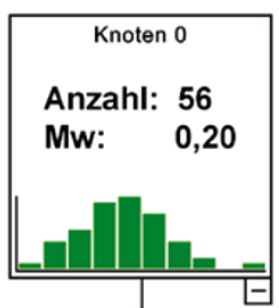

Waldflächenanteil im $5 \mathrm{~km}$ Radius

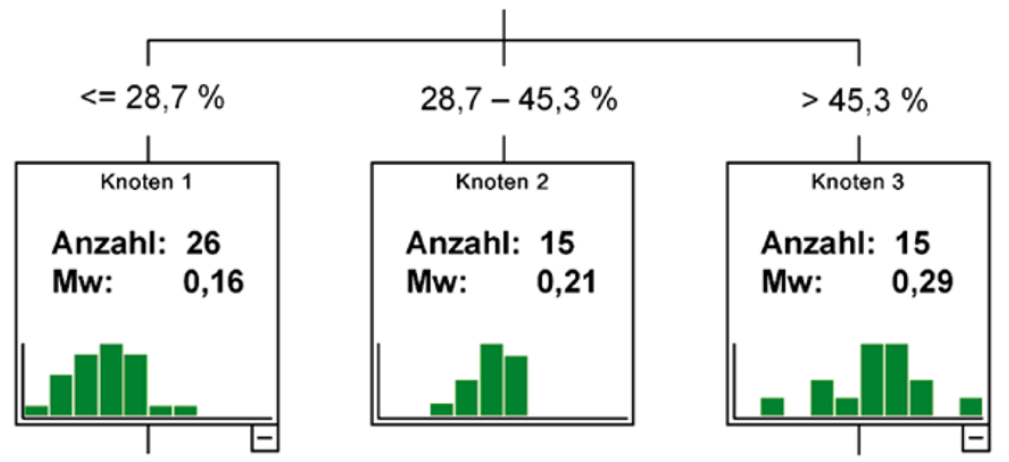

Neigung
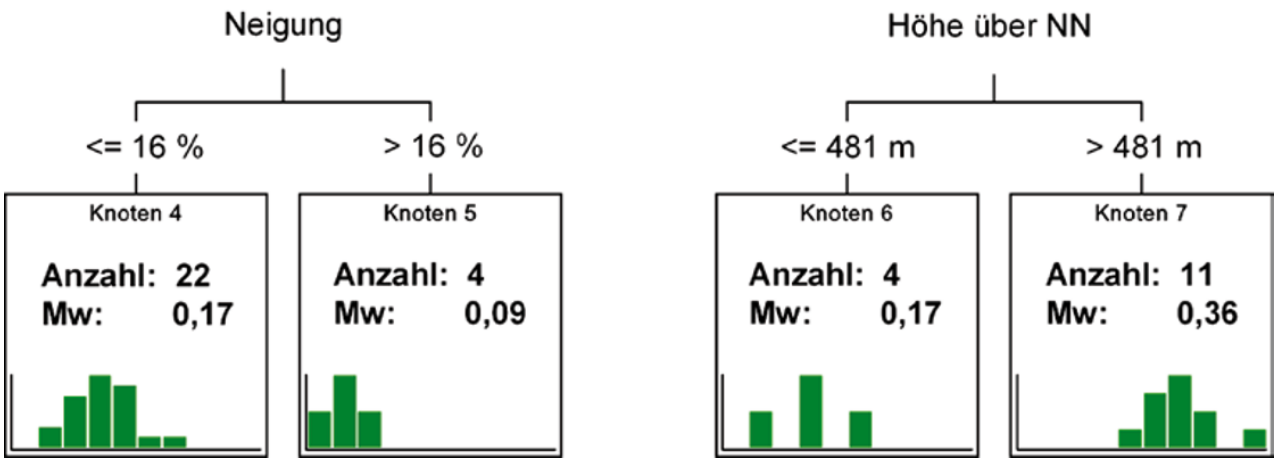

Verbindung stehende (atmosphärische) Verunreinigungen (Bodenschutzkalkungen, Pflegemaßnahmen).

Eine weitere regionaltypische Besonderheit ist darin zu sehen, dass seit 1990 rund 83 bis $97 \%$ der gesammelten und analysierten Moose der Art Hypnum cupressiforme angehörten. Im Bundesgebiet betrugen die Anteile von Hyрnит cupressiforme zwischen 13 und $25 \%$. Wie Siewers et al. (2000) kommen Harmens et al. (2008) zu dem Schluss, dass die Metallakkumulation je nach Metall und Region artenspezifisch sein kann. Eine Umrechnung der Metallkonzentrationen auf jeweils eine Moosart würde Unschärfen erzeugen, gerade bei regional variierenden Metallkonzentrationen. Ferner gestattet es das statistische Design des Moosmonitorings nicht, die mit den verschiedenen Moosarten statistisch assoziierten Metallgehalte zwingend so $\mathrm{zu}$ interpretieren, dass die Unterschiede alleine durch eine unterschiedliche Sammeleffizienz der Moose bedingt ist. Eine solche Interpretation wäre nur dann zulässig, wenn die miteinander verglichenen Metallgehalte von Moosarten stammten, die an jeweils einem Standort gesammelt wurden. Dies ist jedoch nicht der Fall. Eine Umrechnung der von unterschiedlichen Moosen (und Standorten) stammenden Anreicherungswerte auf eine Moosart ist im Falle von Baden-Württemberg nicht erforderlich, da fast nur Proben einer Moosart Gegenstand der Untersuchungen waren. Für überregionale Vergleiche in Deutschland und Europa erscheint eine Umrechnung auch deshalb nicht sinnvoll, weil dann eine Nivellierung räumlich differenzierter Hintergrundwerte erfolgen würde. Die Randbedingungen dieser räumlichen Differenzierung zu identifizieren, sollte vielmehr das Ziel des Umweltmonitorings sein. Im Moosmonitoring gelingt dies flächendeckend, weil in Deutschland und zukünftig auch in anderen europäischen Staaten neben den Messdaten auch wichtige Charakteristika der Moosbeprobungsorte und ihrer Umgebung aufgenommen werden.

Wie für Baden-Württemberg am Beispiel $\mathrm{Pb}$ dargestellt, lässt sich mit dem Moosmonitoring flächendeckend nachweisen, dass abnehmende Metallemissionen und -de- 
positionen in Deutschland zu sinkenden Metallkonzentrationen in Moosen führen. Für EMEP-Depositionsdaten der Metalle $\mathrm{Cd}$ und $\mathrm{Pb}$ lassen sich diese Tendenzen europaweit bestätigen: Cd-Konzentrationen der Gesamtdeposition und in Moosen sind mit $r_{\mathrm{s}}=0,65$ korreliert, bei $\mathrm{Pb}$ sogar mit $r_{\mathrm{s}}=0,73$ (Holy et al. 2010). Bezogen auf das gesamte Bundesgebiet Deutschlands können diese Befunde für einige Orte und Stoffe mit Daten aus Depositionsmessnetzen unterfüttert werden: Bulk-Depositionsdaten stehen im Bundesgebiet für Freiland und Bestand im Falle von $\mathrm{Cd}$ von $n=18$ Standorten, bei $\mathrm{Cu}$ von $n=17$, bei $\mathrm{Pb}$ von $n=19$ und bei Zn von $n=27$ Standorten zur Verfügung. Wet-onlyDepositionsdaten (Freiland) stehen für $\mathrm{As}, \mathrm{Cd}, \mathrm{Cr}$ und $\mathrm{Zn}$ von sechs Standorten des UBA Luftmessnetzes und des ICP Integrated Monitoring zur Verfügung (Schröder et al. 2009). Die Rangkorrelationen zwischen den Metallkonzentrationen in Moosen einerseits und diesen Depositionsdaten sowie modellierter Nass-, Trocken- und Gesamtdeposition andererseits variieren nach Stoffen und Depositionsmechanismus. Unter Berücksichtigung der niedrigen Anzahl von Vergleichsmessungen lassen sich hohe und sehr hohe Korrelationen zwischen den Konzentrationen von As und $\mathrm{Cr}$ in Moosen und in der Wet-only-Deposition nachweisen. Moderate Korrelationen weisen die Konzentrationen von $\mathrm{Cd}, \mathrm{Cu}, \mathrm{Pb}$, and $\mathrm{Zn}$ in Moosen und Depositionen auf. Die statistischen Beziehungen zwischen den Konzentrationen in Bestandsdepositionen und Moosen sind bei $\mathrm{Cd}, \mathrm{Cu}$ and $\mathrm{Pb}$ gering, mittelstark hingegen für $\mathrm{Zn}$. Die Korrelationen von $\mathrm{Cd}$ and $\mathrm{Pb}$ in Moosen und den modellierten Depositionen sind niedrig bis mittel und variieren zeitlich, stoffspezifisch und nach modelliertem Depositionsprozess: Die höchsten entsprechenden Korrelationen bestehen zwischen den Konzentrationen von $\mathrm{Cd}$ und $\mathrm{Pb}$ in Moosen und in der Gesamtund Trockendeposition (Schröder et al. 2009).

Weitere Korrelationsanalysen zeigen, dass die statistischen Beziehungen zwischen den in den Moosen akkumulierten Metallen und Stickstoff sowie Eigenschaften der Beprobungsorte und ihrer Umgebung plausibel und niedrig bis stark ausgeprägt sind. Die räumliche Differenzierung der Metallanreicherungen ist wesentlich mit den Depositionen sowie folgenden lokalen und regionalen Gegebenheiten korreliert: Traufeffekt, Landnutzung (Flächenanteile Wald, Landwirtschaft, bebaute Flächen) und Höhe über NN.

\section{Schlussfolgerungen}

Umweltmonitoring ist Kernbestandteil internationaler Nachhaltigkeitsstrategien (UNESCO MaB, UNECE ICP Vegetation). Um Umweltveränderungen zuverlässig aufzeigen zu können, bedarf es langfristig betriebener Messnetze. Das Moosmonitoring ist ein Langfristmessnetz des ICP Vegetation. Während physikalische Messnetze (z.B.
UBA Luftmessnetz, EMEP) nur an wenigen Orten Daten erheben können, liefert das Moosmonitoring räumlich hoch aufgelöste Daten, die für die räumliche Modellierung der Depositionen und die räumlich differenzierte Erfassung und Bewertung von Stoffanreicherungen in der Umwelt unentbehrlich sind. Im Gegensatz zu Depositionsmessungen gibt das Moosmonitoring Auskunft darüber, welche Stoffe und in welcher Höhe am Wirkort/Schutzgut Pflanze (Moos) ankommen. Die Bioakkumulation von Stoffen ist für die ökotoxikologische Bewertung von Stoffeinträgen z. B. in Naturschutzgebieten und FFH-Gebieten aussagekräftiger als die gemessene atmosphärische Deposition. Das Moosmonitoring liefert also nicht nur die Bestimmung der Umweltkonzentration von Stoffen, sondern immissionsschutzrechtlich relevante Beiträge zur Wirkungsermittlung, denn die Stoffanreicherung in einem Organismus ist die Vorstufe einer potenziellen physiologischen Wirkung. Insofern ist das passive Expositionsmonitoring mit Moosen insbesondere im Hinblick auf Vorsorgemaßnahmen wichtig. Das Moosmonitoring ist ein wichtiges Bindeglied zwischen technischen Depositionsmessungen und biologischen Wirkungen. Ein Schwermetallindikator auf Grundlage der Moosmonitoringdaten liegt für einzelne Naturräume, Bundesländer und Schutzgebiete vor.

\section{Empfehlungen und Ausblick}

Das Moosmonitoring sollte als Langfristmonitoring fortgeführt und zur Erfassung und Bewertung des Eintrags und der Anreicherung von Stoffen in Schutzgebiete genutzt werden. Zudem empfiehlt es sich, das Moosmonitoring enger mit dem Humanbiomonitoring (HBM) zu verknüpfen und damit einen wichtigen Beitrag zum Programm Transport Health and Environment Pan European Programme (THE PEP) von UNECE und WHO-Europe zu leisten (http:// www.thepep.org/en/welcome.htm). Im HBM fehlt insbesondere die räumlich differenzierte Erfassung der Beziehungen zwischen der inneren Exposition von Probanden durch Humanbiomonitoring einerseits sowie der äußeren Exposition innerhalb und außerhalb von Gebäuden durch Umweltmonitoring andererseits (Pesch et al. 2009; Zechmeister et al. 2007).

\section{Literatur}

Bealey WJ, Cape JN, Leith ID, Long S, Kinnerlsey RP (2008a) Air quality outcomes in pollution regulation: strengths, limitations and potential. Science Report SC030175/SR1, CEH Project Number: C02600. Environment Agency, Bristol, S 1-47

Bealey WJ, Long S, Spurgeon DJ, Leith I, Cape JN (2008b) Review and implementation study of biomonitoring for assessment of air quality outcomes. Science Report SC030175/SR2. Environment Agency, Bristol, S 1-170 
Broecker F, Schmidt G, Schröder W (2007) Medienübergreifende Untersuchung zur Versauerung in Baden-Württemberg. In: Wichmann H-E, Schlipköter H-W, Füllgraf G (Hrsg) Handbuch der Umweltmedizin. ecomed, Landsberg am Lech, Kap IV/7.1, S 17-19

Dämmgen U (2005) Bestimmung von Ammoniak-Einträgen aus der Luft und deren Wirkungen auf Waldökosysteme. ANSWER-Projekt, Landbauforschung Völkenrode, Braunschweig, Sonderheft 279, S 1-113

Forster E-M, Matthies M, Brüggemann R (1993) Optimierung eines Bioindikator-Meßnetzes mit geostatistischen Methoden und einem geographischen Informationssystem. Umweltwiss Schadst Forsch 5:286-294

Funk W, Dammann V, Donnevert G (2006) Quality assurance in analytical chemistry. Applications in environmental, food and materials analysis, biotechnology and medical engineering, 2 Aufl. Wiley-VCH, Weinheim

Genßler L, Rademacher J, Rammert U (2001) Arbeitskreis der Landesanstalten und -ämter Konzeption der künftigen Aufgabenbereiche. Umweltwiss Schadst Forsch 13:1-4

Gigon A, Grimm V (1997) Stabilitätskonzepte in der Ökologie: Typologie und Checkliste für die Anwendung. In: Fränzle O, Müller F, Schröder W (Hrsg) Handbuch der Umweltwissenschaften. ecomed, Landsberg, Kap III/2.3, S 1-19

Harmens H (2005) Monitoring of atmospheric deposition in Europe using bryophytes. Monitoring manual 2005/2006 survey, Bangor, UK

Harmens H, Norris D and the participants of the moss survey (2008) Spatial and temporal trends in heavy metal accumulation in mosses in Europe (1990-2005). Programme Coordination Centre for the ICP Vegetation, Centre for Ecology and Hydrology, Environment Centre Wales, Bangor, UK

Herpin U, Siewers U, Markert B, Rosolen V, Breulmann G, Bernoux M (2004) Second German heavy-metal survey by means of mosses, and comparison of the first and second approach in Germany and other European countries. Environ Sci Pollut Res 11:57-66

Holy M, Pesch R, Schröder W, Ilyin I, Harmens H, Alber R, Aleksiayenak Y, Blum O, Coskun M, Dam M, de Temmermann L, Frolova M, Frontasyeva M, Grodzińska K, Jeran Z, Kubin E, Kvietkus K, Leblond S, Liiv S, Magnússon S, Maňkovská B, Miodrag K, Rühling Å, Santamaria J, Steinnes E, Suchara I, Szymon K, Thöni L, Urumov V, Yurukova L, Zechmeister (2010) First thorough identification of factors associated with $\mathrm{Cd}, \mathrm{Hg}$ an $\mathrm{Pb}$ concentrations in mosses sampled in the European Surveys 1990, 1995, 2000, and 2005. J Atmos Chem, DOI 10.1007/s10874-010-9160-3

Johnston K, Ver Hoef JM, Krivoruchko K, Lucas N (2001) Using ArcGIS Geostatistical Analyst. Redlands

Kass GV (1980) An exploratory technique for investigating large quantities of categorial data. Appl Stat 29:199-127

Keil M, Kiefl R, Strunz G (2005) CORINE Land Cover 2000 - Germany. Final Report. German Aerospace Center, German Remote Sensing Data Center, Oberpfaffenhofen

Kleppin L, Schröder W, Pesch R, Schmidt G (2008) Entwicklung und Erprobung einer Metadaten- und WebGIS-Applikation für das Expositionsmonitoring mit Moosen in Deutschland. Ein Beitrag zum LTER-Netzwerk. Umweltwiss Schadst Forsch 20:38-48

Kostka-Rick R, Leffler US, Markert B, Herpin U, Lusche M, Lehrke J (2001) Biomonitoring zur wirkungsbezogenen Ermittlung der Schadstoffbelastung in terrestrischen Ökosystemen. Konzeption, Durchführung und Beurteilungsmaßstäbe im Rahmen von Genehmigungsverfahren. Umweltwiss Schadst Forsch 12:5-12

LfU (Landesanstalt für Umweltschutz Baden-Württemberg, Hrsg.) (2003) Medienübergreifende Umweltbeobachtung. Stand und Perspektiven. Karlsruhe

LUBW (Landesanstalt für Umwelt, Messungen und Naturschutz Baden-Württemberg, Umweltministerium Baden-Württemberg, Hrsg.) (2009) Umweltdaten 2009 Baden-Württemberg. Karlsruhe Stuttgart
Matheron G (1965) Les variables régionalisées et leur estimation. Masson, Paris

Mohr K (1999) Passives Monitoring von Stickstoffeinträgen in Kiefernforsten mit dem Rotstengelmoos (Pleurozium schreberi (Brid) Mitt). Umweltwiss Schadst Forsch 11:267-274

Mohr K (2007) Biomonitoring von Stickstoffimmissionen. Möglichkeiten und Grenzen von Bioindikationsverfahren. Umweltwiss Schadst Forsch 19(4):255-264

Pesch R (2003) Geostatistische und multivariat-statistische Analyse des Moos-Monitorings 1990, 1995 und 2000 zur Ableitung von Indikatoren für die Bioakkumulation atmosphärischer Metalleinträge in Deutschland. Dissertation, Hochschule Vechta

Pesch R, Schmidt G, Schröder W, Aden C, Kleppin L, Holy M (2007a) Development, implementation and application of the WebGIS MossMet. In: Tochtermann K, Scharl A (Hrsg) The geospatial web. How geo-browsers, social software and the Web 2.0 are shaping the network society. London, Springer, S 191-200

Pesch R, Schröder W, Genßler L, Göritz A, Holy M, Kleppin L, Matter Y (2007b) Moos-Monitoring 2005/2006: Schwermetalle IV und Gesamtstickstoff. Umweltforschungsplan des Bundesministers für Umwelt, Naturschutz und Reaktorsicherheit. FuE-Vorhaben 205 64200, Abschlussbericht, im Auftrag des Umweltbundesamtes, Berlin

Pesch R, Schröder W, Dieffenbach-Fries H, Genßler L, Kleppin L (2008) Optimierung des Moosmonitoring-Messnetzes in Deutschland. Umweltwiss Schadst Forsch 20:49-61

Pesch R, Schmidt G, Schröder W, Conrad A, Kolossa-Gehring M, Feigenspan S, Utermann J (2009) Das Potenzial raumbezogener Daten im Human-Biomonitoring am Beispiel des KinderUmwelt-Surveys. In: Strobl J, Blaschke T, Griesebner G (Hrsg) Angewandte Geoinformatik 2009, Wichmann, Heidelberg, S 248-257

Rüdel H, Bester K, Eisenträger A, Franzaring J, Haarich $M$, Köhler J, Körner W, Oehlmann J, Paschke A, Ricking M, Schröder W, Schröter-Kermani C, Schulze T, Schwarzbauer J, Theobald N, Von der Trenck, T, Wagner G, Wiemüller GA (Arbeitskreis Umweltmonitoring in der GDCh-Fachgruppe Umweltchemie und Ökotoxikologie) (2007) Stoffbezogenes Umweltmonitoring. In: Wichmann H-E, Schlipköter H-W, Füllgraf $\mathrm{G}$ (Hrsg) Handbuch der Umweltmedizin. ecomed, Landsberg, Kap. IV/7.1, S 1-48

Rühling A, Tyler G (1968) An ecological approach to the lead problem. Bot Notiser 121:321-343

Rühling A, Tyler G (1969) Ecology of heavy metals - a regional and historical study. Bot Notiser 121:248-259

Rühling A, Tyler G (1970) Sorption and retention of heavy metals in the woodland moss Hylocomium splendens (Hedw.) Br Et Sch Oikos 21:248-342

Schröder W, Schmidt G (2000) Raumgliederung für die Ökologische Umweltbeobachtung des Bundes und der Länder. Umweltwiss Schadst Forsch 12:237-243

Schröder W, Garbe-Schönberg CD, Fränzle O (1991) Die Validität von Umweltdaten Kriterien für ihre Zuverlässigkeit: Repräsentativität, Qualitätssicherung und -kontrolle. Umweltwiss Schadst Forsch 3:237-241

Schröder W, Pesch R, Schmidt G (2004) Soil monitoring in Germany Spatial representativity and methodical comparability. J Soils Sediments 4:49-58

Schröder W, Schmidt G, Hasenclever J (2005) Korrelation meteorologischer und phänologischer Indikatoren des Klimawandels? Statistische Analyse am Beispiel Baden-Württembergs. UWSF/Z Umweltchem Ökotoxikol 17(2):94-105

Schröder W, Schmidt G, Zipperle J (2006) Geodaten, Messdaten und Analyseabläufe zur Messflächenauswahl bei unterschiedlichen Skalen. Überlegungen zum GVO-Messnetz in Baden-Württemberg. BfN-Skripten 189:29-41 
Schröder W, Englert C, Pesch R, Zechmeister HG, Thöni L, Suchara I, Maňkovská B, Jeran Z, Harmens H, Grodzinska K, Alber R (2008) Metallakkumulation in Moosen: Standörtliche und regionale Randbedingungen des Biomonitoring von Luftverunreinigungen. Umweltwiss Schadst Forsch 20:120-132

Schröder W, Pesch R, Matter Y, Görlitz A, Dieffenbach-Fries H, Genßler L (2009) Trend der Schwermetall-Bioakkumulation 1990 bis 2005: Qualitätssicherung bei Probenahme, Analytik, geostatistischer Auswertung. Umweltwiss Schadst Forsch 21(6):549-574

Siewers U, Herpin U, Straßburger S (2000) Schwermetalleinträge in Deutschland Moos-Monitoring 1995 Teil 2. Geologisches Jahrbuch, Sonderhefte, Heft SD 3, Stuttgart, Bornträger

Steinnes E, Rühling Å, Lippo H, Mäkinen A (1997) Reference materials for large-scale metal deposition surveys. Accredit Qual Assur 2(5):243-249

Tyler G (1990) Bryophytes and heavy metals: a literature review. Bot J Linnean Soc 104:231-253
Wappelhorst O, Korhammer S, Leffler US, Markert B (2000) Ein Moosbiomonitoring zur Ermittlung atmosphärischer Elementeinträge in die Euroregion Neiße (D, PL, CZ) UWSF/Z Umweltchem Ökotox 12(4):191-200

Webster R, Oliver MA (2001) Geostatistics for environmental scientists. Wiley, Chichester New York Weinheim Brisbane Singapore Toronto

Zechmeister HG, Dullinger S, Hohenwallner D, Riss A, Hanus-Illnar A, Scharf S (2006) Pilot study on road traffic emissions (PAHs, heavy metals) measured by using mosses in a tunnel experiment in Vienna, Austria. Environ Sci Pollut Res 13:398-405

Zechmeister HG, Köllensperger G, Hann S, Hanus-Illnar A, Scharf S, Schneider J, Neuberger M, Moshammer H, Hohenwallner D, Schierl R, Schröder W, Schmidt G, Pirintsos SA, Loppi S, Gaggi C, Nante N, Namiesnik J, Zygmunt B, Jeran Z, Perharic L, Künzli N, Frontasyeva M, Lyapunov S, Ilchenko I, Harmens H (2007) INDOMO. New tool for monitoring indoor pollutants with health relevance across Europe. Wien 